\title{
Small is beautiful
}

The Small GTPase Ran

edited by Mark Rush and Peter D'Eustachio

Kluwer Academic Publishers, 2002

Hardback $\bullet £ 91 / \$ 130$

Paul Clarke

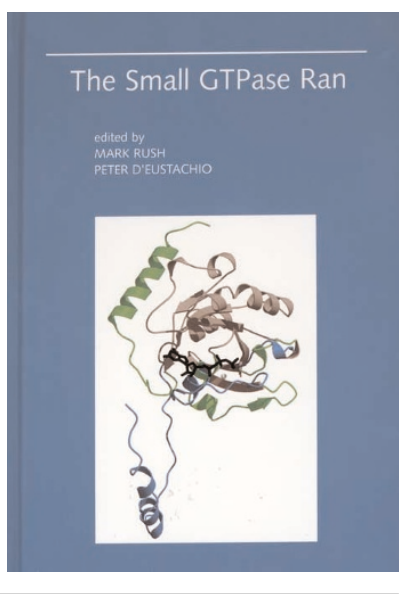

0 ne of the joys of cell biology is the unexpected, the function that cannot be readily predicted from form alone. Over the last dozen years, since it was identified as a Ras-related cDNA by Rush, D'Eustachio and colleagues and purified by Bischoff and Ponstingl as a mainly nuclear GTPase, Ran has emerged as a central regulator in the organization of eukaryotic cells. Like other members of Ras superfamily of small GTPases, Ran controls the assembly of multiprotein complexes by switching between GTP- and GDP-bound states. Ran is unusual as it lacks amino- or carboxy-terminal modifications, which anchor related GTPases to cellular membranes. In fact, it is probably highly mobile, although it is concentrated in the nucleus during interphase. Nevertheless, like other GTPases, its biological function may also be determined by its localization to specific structures, particularly during mitosis. In essence, it seems that Ran works by distinguishing the molecular environment of the nucleus from the cytoplasm, a role that has profound implications for the evolution of eukaryotes.

To date, Ran has been perhaps underrepresented in the hardback literature, yet the general importance of the mechanisms in which Ran is central means that this GTPase is likely to be of interest to many cell biologists and biochemists. For the first time, Mark Rush and Peter D'Eustachio have brought together many of the acknowledged experts on Ran in a single volume. Each of the ten chapters can be read alone and the references are remarkably up-to-date, an important consideration for a book covering such a rapidly evolving area of research. Generally, the chapters are well written and concise, providing a scholarly review of the current state of research in an area of cell biology or biochemistry in which Ran is involved. Inevitably, there is some overlap between the chapters, but this allows for a useful comparison. For the specialist, the most interesting parts of the book are where the

\section{Each of the ten chapters can \\ be read alone and the \\ references are remarkably \\ up-to-date, an important \\ consideration for a book \\ covering such a rapidly \\ evolving area of research.}

authors have indulged in some speculation and even described unpublished work, but non-specialist may have difficulty in critically assessing the more speculative ideas. Much of the work on Ran has yet to reach the student texts, while no single review article can cover all of the accumulated results. This volume fills this gap admirably and is likely to be an essential handbook for laboratories working on Ran. Researchers whose interests include processes in which Ran is involved - for instance, those studying nucleocytoplasmic transport, mitosis or nuclear structure - will also find much of interest here.

To date, the progress of research on Ran illustrated in this book has been advanced by a variety of different model organisms and cell-free systems backed up by good biochemistry. The functions of Ran in the transport of proteins and RNA species between the nucleus and the cytoplasm are covered in the first four chapters, authored by Mary Shannon Moore (who discovered the function of Ran in nuclear transport), Bryce Paschal and Catherine Dargemont (CRM1-dependent nuclear export pathway), Anita Hopper (RNA export in yeast) and Elsebet Lund and James Dahlberg (RNA export in higher eukaryotes). Andrew Wilde discusses the more recently identified role of Ran in mitotic spindle assembly, a function that has relied heavily on the use of mitotic Xenopus laevis egg extracts, in which spindles can be assembled in vitro. In a later chapter, Patrizia Lavia and colleagues discuss the possible role of the Ran system during mitosis in somatic mammalian cells. The recently identified role of Ran in directing nuclear envelope formation is not addressed in a specific article, but the initial discoveries are covered in two chapters that discuss the multiple roles of Ran during the cell division cycle in higher eukaryotes (Mary Dasso and colleagues) and fission yeast (Sandra Salus and Shelley Sazer). The biochemistry of critical regulators and effectors of Ran is described by Ralf Bischoff and Herwig Ponstingl in the penultimate chapter, while Klaus Scheffzek and Alfred Wittinghofer review the remarkable progress that has been made in understanding the molecular structures of Ran and its partners. Each chapter ends with a useful summary and the book is prefaced by the editors with an overview of the Ran system. However, a summary of the nomenclature of the Ran system, particularly the importin/exportin family of interacting proteins, and a comparison of yeast and vertebrate names, would have provided a valuable resource.

Research on the cell biology of the Ran system is a particularly attractive field because it is increasingly hypothesis-driven and generates exciting new ideas about the organization of cells. Graduate students and postdoctoral researchers who are looking for an active and expanding area of research that provides an exciting intellectual challenge will find this book an excellent place to start. Our understanding of the Ran system is only in its infancy and a follow-up book of the next decade's research is likely to be a much weightier volume. Paul Clarke is in the Biomedical Research Centre, University of Dundee, Ninewells Hospital and Medical School, Dundee, Scotland, DD1 9SY, UK email:paul.clarke@cancer.org.uk 